\title{
There is no special condition where a velocity of a light wave is invariance in all inertial frames.
}

Mahesh Gupta ( $\square$ truthofphysics@gmail.com )

Madurai Kamaraj University https://orcid.org/0000-0002-1772-8490

Prafful Acharya

Amrita Vishwa Vidyapeetham University

Rampravesh Yadav

University of Mumbai

Article

Keywords: Frame of Reference, The Time, Newton's Law of motion, Galilean Transformation, Linear Motion, Periodic Motion, Particle-Wave Duality of Light, Lorentz Transformation, Special Theory of Relativity

Posted Date: January 15th, 2021

DOI: https://doi.org/10.21203/rs.3.rs-143936/v2

License: (c) (i) This work is licensed under a Creative Commons Attribution 4.0 International License. Read Full License 


\title{
There is no special condition where a velocity of a light wave is invariance in all inertial frames.
}

\author{
Mahesh Gupta, Prafful Acharya \& Rampravesh R. Yadav \\ truthofphysics@gmail.com \\ Mumbai, Maharashtra, India
}

\begin{abstract}
:
This study of the literature reveals that the concept such as the velocity of a light wave is invariance in all inertial frames is wrong. A velocity of any motion always depends upon its reference frame. There is no special condition present in nature where the velocity is invariance in all inertial frames. Galileo Galilei defined speed is the distance covered per unit of time. Sir Isaac Newton defined velocity is the speed with direction. In the same period, physicists introduced the concept such as wavelength and frequency for measuring the speed a wave. The wave motion in which cycles are repeating after the same interval of time with the same distance between them. In 1887, Albert A. Michelson and Edward W. Morley performed an experiment to detect ether medium. But they failed to prove the existence of the ether medium and published Null Result. This Null Result has led to the creation of various new hypothetical theories. This study tries to reveal the relation between mathematics and physics and also comprehensive literature of light is a particle-wave duality in nature.
\end{abstract}

Keywords: Frame of Reference, The Time, Newton's Law of motion, Galilean Transformation, Linear Motion, Periodic Motion, Particle-Wave Duality of Light, Lorentz Transformation, Special Theory of Relativity.

\section{Introduction:}

Galileo Galilei defined speed is the distance covered per unit of time [1]. Sir Isaac Newton defined velocity is the speed with direction [2]. From 17th century, physicists introduced the concept such as wavelength $(\lambda)$ and frequency (f) for measuring the speed of a wave. The wave motion in which cycles are repeating after the same interval of time with the same distance between them. Earlier physicists believe that if an event propagates in the form of a wave in nature, then it required medium. Like sound wave and water wave. After James Clerk Maxwell demonstrated that the light is the part of electromagnetic radiation. Which are synchronized oscillations of electric and magnetic fields in nature [3]. Then physicists were trying to find an electromagnetic synchronized medium called "ether medium" [3].

In 1887, Albert A. Michelson and Edward W. Morley performed an experiment to detect ether medium. But they failed to prove the existence of the ether medium and published Null Result. Where Michelson-Morley's mathematical calculation and observation result were always different [4]. This was mysterious to the scientific community at that the time and created various new theories to explain this difference between mathematical result and observation result or the Null result.

The First Length Contraction idea was given by George FitzGerald (1889). Later it formalized by Hendrik Lorentz (1892) by working on the invariance of 'c' [6]. In 1905, Albert Einstein framing a special theory of relativity by working on Lorentz Transformation. The special theory of relativity in which Length contraction and Time dilation like hypothesis terms used to fix this difference and gave explanation [7].This causing confusion or misunderstanding for knowledge about Nature. Their theories deceive by producing a misleading or false impression of reality.

\section{Rest or Uniform motion in a straight line (velocity):}

An object at rest or uniform motion in a straight line totally depends upon its observer. If Observer says an object at rest or uniform motion in a straight line then observer specifies the frame of reference. 
Newton's 1st law of motion: "Every object will remain at rest or in uniform motion in a straight line unless an unbalanced force act upon it." [2]

Speed: It is defined as an object covered by distance with respect to time. It is denoted by 's' and the mathematical equation is

$$
s=\frac{d}{t}
$$

Velocity: It is defined as the speed of an object with required direction. It is denoted by ' $v$ ' and the mathematical equation is same as speed but it required direction.

$$
\vec{v}=\frac{d}{t}
$$

Reference Frame: When we say an object is moving, this displacement with respect to its observer and he creates his frame this is known as the reference frame. Generally, we have seen all events on earth and we take the same reference frame for all observers on the earth that is the ground frame. The frame of an observer is not an absolute quantity in nature.

Time: It is our changing measurement scale. The time takes for any events to happen the same for all observers in nature. Time is an absolute quantity in nature.

Wave property of ' $c$ ': It is the product of the numbers of repeating cycles per unit time and the distance between them in a wave motion. It is denoted by ' $c$ ' and the mathematical equation is

$$
c=\lambda \times f
$$

Where,

$\lambda=$ the distance between any two adjacent event in wave motion.

$\mathrm{f}=$ the numbers of repeating cycles per unit time in wave motion

Relative Motion: In which observer observed many events in one frame of reference and find out relative velocity between them.

Relative Observation: In which observers observed the same event in a relative frame of reference and give mathematics in their own reference frame.

\section{Analysis of uniform motion in a straight line with in relative frame of reference:}

a) Consider the $1^{\text {st }}$ observer ' $A$ ' observed this event and in its reference frame ' $M$ ':

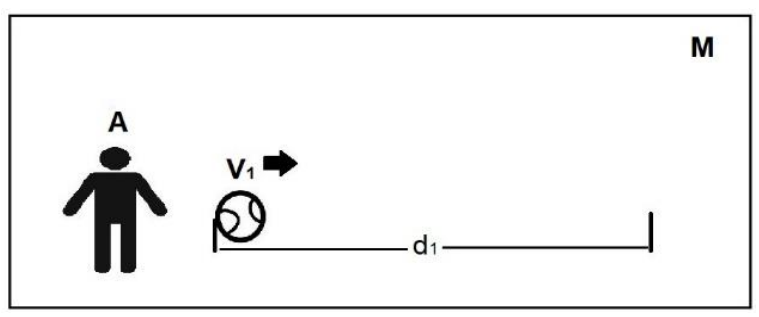

Figure 2.1 
Observer ' $A$ ' observed particle which travels with velocity in positive $\mathrm{x}$-direction in its reference frame ' $\mathrm{M}$ '. Let's take velocity ' $V_{1}$ ' and reach a distance ' $d_{1}$ ' in time ' $t$ ' for observer ' $A$ ' in its reference frame ' $M$ '. (Figure 2.1)

Eq. $\overrightarrow{\mathrm{V}}_{1}=\left(\mathrm{d}_{1}\right) / \mathrm{t}$, (particle velocity for observer 'A')

$\left(\mathrm{V}_{1}\right) \times \mathrm{t}=\mathrm{d}_{1}$ (distance reached by particle in time ' $\mathrm{t}$ ' for observer ' $\mathrm{A}$ ')

b) Observer ' $A$ ' is moving with velocity $V_{2}$ in positive $x$ - direction with respect to $2^{\text {nd }}$ observer ' $B$ ' in its reference frame ' $N$ '.

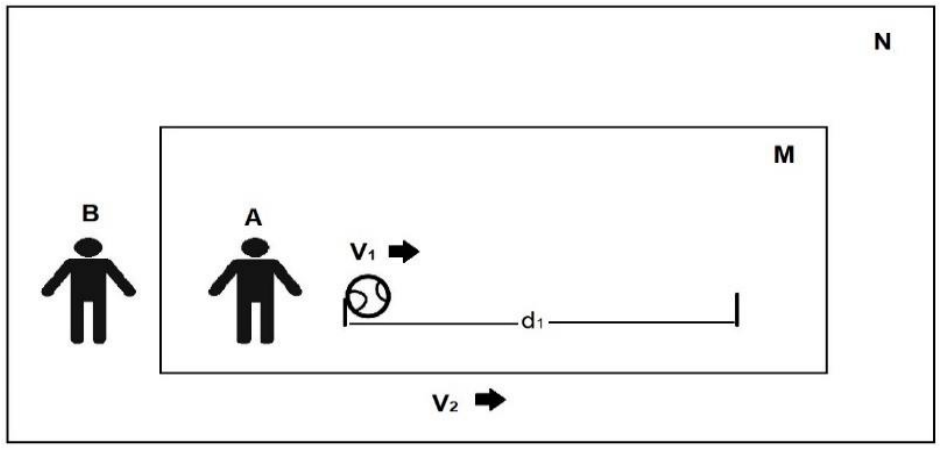

Figure 2.2

There is another observer (B), who is observing this phenomenon its reference frame ' $N$ '. Observer (A) is travelling with a velocity of ' $V_{2}$ ' with respect to observer ' $B$ '. For observer ' $B$ ', the same particle reaches a distance of ' $\mathrm{d}_{2}$ ' at in same time with a velocity of ' $\mathrm{V}_{3}$ ' and the direction of the particle is the same in its reference frame ' $\mathrm{N}$ '. (Figure 2.2)

Eq. $\overrightarrow{\mathrm{V}_{3}}=\overrightarrow{\mathrm{V}_{1}}+\overrightarrow{\mathrm{V}_{2}}$

(particle velocity for observer 'B')

$\left(\mathrm{V}_{3}\right) \mathrm{t}=\left(\mathrm{V}_{1}\right) \mathrm{t}+\left(\mathrm{V}_{2}\right) \mathrm{t}$

(distance reached by particle in time ' $\mathrm{t}$ ' for observer ' $\mathrm{B}$ ')

$\mathrm{d} 3=\mathrm{d} 1+\mathrm{d} 2$

c) If particle velocity ' $V_{1}$ ' travelling in negative $x$-direction with respect to observer ' $A$ ' in its reference frame ' $M$ ' and observer ' $A$ ' is travelling with velocity ' $V_{2}$ ' in positive $\mathrm{x}$-direction with respect to observer ' $\mathrm{B}$ ' in its reference frame 'N'. (Figure 2.3)

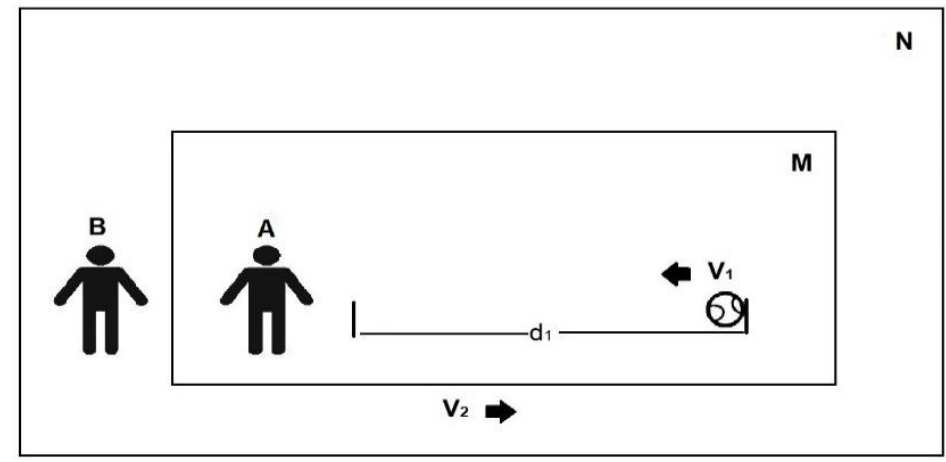

Figure 2.3

Then observer ' $B$ ' observed the velocity of particle ' $V_{3}$ ' is reached distance ' $d_{3}$ ' in same time ' $t$ ' in its reference frame 'N'.

i. If ' $\mathrm{V}_{1}$ ' $>$ ' $\mathrm{V}_{2}$ ' then observer ' $\mathrm{B}$ ' observed the particle is travelling in negative $\mathrm{x}$ - direction.

Eq. $\mathrm{V}_{3}=\mathrm{V}_{1}-\mathrm{V}_{2}$ (velocity of particle for observer 'B')

$\mathrm{d}_{3}=\mathrm{d}_{1}-\mathrm{d}_{2}$ (distance reached by particle in same time ' $t$ ' for observer ' $\mathrm{B}$ ') 
ii. If ' $\mathrm{V}_{1}$ ' $<$ ' $\mathrm{V}_{2}$ ' then observer ' $\mathrm{B}$ ' observed the particle is travelling in positive $\mathrm{x}$ - direction.

Eq. $\overrightarrow{\mathrm{V}}_{3} \stackrel{\leftarrow}{=} \mathrm{V}_{1}-\mathrm{V}_{2} \quad$ (velocity of particle for observer ' $\mathrm{B}$ ')

$\mathrm{d}_{3}=\mathrm{d}_{1}-\mathrm{d}_{2} \quad$ (distance reached by particle in same time ' $\mathrm{t}$ ' for observer ' $\mathrm{B}$ ')

iii. If ' $\mathrm{V}_{1}{ }^{\prime}={ }^{\prime} \mathrm{V}_{2}$ ' then observer ' $\mathrm{B}$ ' observed the particle is in rest position.

Eq. $\mathrm{V}_{3} \stackrel{\longleftarrow}{=} \overrightarrow{\mathrm{V}_{1}}-\overrightarrow{\mathrm{V}_{2}}=0 \quad$ (velocity of particle for observer ' $\mathrm{B}$ ')

$\mathrm{d}_{3}=\mathrm{d}_{1}-\mathrm{d}_{2} \quad$ (distance reached by particle in same time ' $\mathrm{t}$ ' for observer ' $\mathrm{B}$ ')

d) If particle travelling with velocity ' $\mathrm{V}_{1}$ ' in the positive $y$-direction with respect to observer ' $A$ ' (Figure 2.4) and observer ' $A$ ' travelling with velocity ' $\mathrm{V}_{2}$ ' in positive $\mathrm{x}$-direction with respect to observer ' $\mathrm{B}$ '. (Figure 2.5)

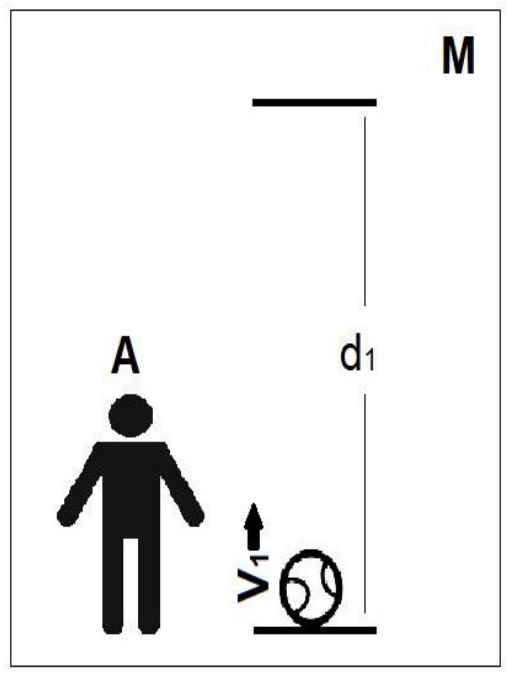

Figure 2.4

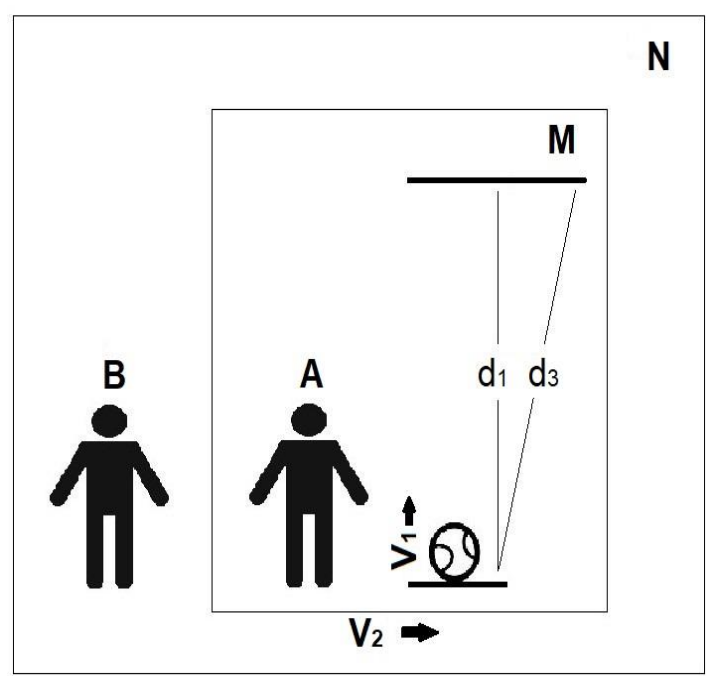

Figure 2.5

Then observer ' $B$ ' observed particle velocity ' $V_{3}$ ' is reached distance ' $d_{3}$ ' same in time ' $t$ '.

Eq. $\quad\left(\overrightarrow{\left.\mathrm{V}_{3}\right)^{2}}=\overrightarrow{\left(\mathrm{V}_{1}\right)^{2}}+\overrightarrow{\left(\mathrm{V}_{2}\right)^{2}}\right.$

(by Pythagoras theorem)

$\left(d_{3}\right)^{2}=\left(d_{1}\right)^{2}+\left(d_{2}\right)^{2}$

e) If particle travelling with velocity ' $\mathrm{V}_{1}$ ' in negative $\mathrm{y}$-direction with respect to observer ' $\mathrm{A}$ ' in its reference frame ' $M$ ' (Figure 2.6) and observer ' $A$ ' travelling with velocity ' $V_{2}$ ' in positive $x$-direction with respect to observer ' $B$ ' in its reference frame 'N'. (Figure 2.7)

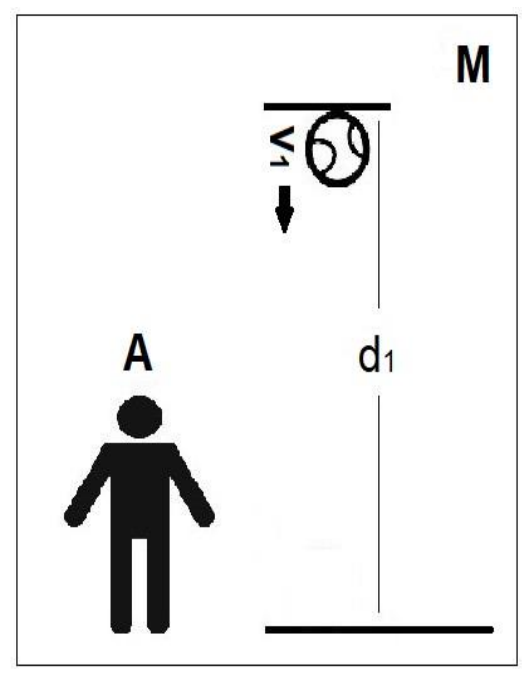

Figure 2.6

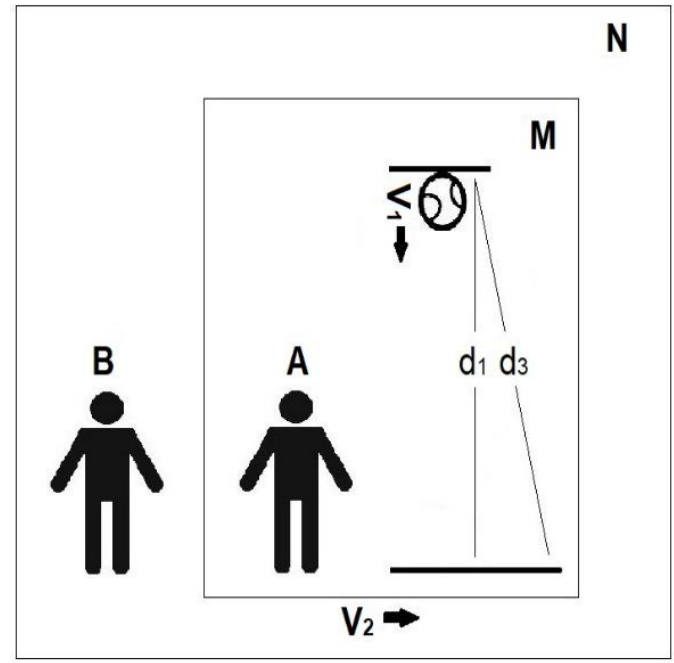

Figure 2.7 
Then observer ' $B$ ' observed particle velocity ' $V_{3}$ ' is reached distance ' $d_{3}$ ' in same time ' $t$ '.

Eq. $\quad\left(\overrightarrow{\mathrm{V}_{3}}\right)^{2}=\left(\overrightarrow{\mathrm{V}_{1}}\right)^{2}+\left(\overrightarrow{\mathrm{V}_{2}}\right)^{2}$

(by Pythagoras theorem)

$$
\left(d_{3}\right)^{2}=\left(d_{1}\right)^{2}+\left(d_{2}\right)^{2}
$$

Therefore,

$\mathrm{V}_{1}=$ velocity of particle for observer ' $A$ ' in its reference frame ' $M$ '.

$\mathrm{V}_{2}=$ velocity of observer ' $\mathrm{A}$ ' respect to observer ' $\mathrm{B}$ '.

$\mathrm{V}_{3}=$ velocity of particle for observer ' $\mathrm{B}$ ' in its reference frame ' $\mathrm{N}$ '.

\section{$1^{\text {st }}$ mistake:}

If we talking about the speed of a motion. That means we observed a motion in only one frame of reference. When we trying to solve problems in its relative frames. Then it is necessary to define the direction of the motion. The postulate such as the speed of light is constant in all inertial frames is itself untenable to solve problems in its relative inertial frames.

\section{Prove:}

If you are to travel by train. Where the train is travelling with constant velocity. Then you observed all the objects within a train at rest with respect to you. But if you see outside of the train, then you observed all objects are moving with respect to you. At the same time, your friend is standing on the ground. Then he observed you and all objects within a train are moving with respect to him. This is the too simple Experimental observation which you observed in daily life to prove a displacement and velocity of an object always depends upon its frame of reference.

\section{Conclusion:}

- The displacement of a motion always depends upon its frame. That means the displacement of a motion is not the same in all inertial frames.

- The frame created by an observer for measuring the displacement of any motion in its frame. Therefore, The frame is not an absolute quantity in nature.

- The time takes for any events to happen the same for all observers in nature.

- That's the reason the velocity of a motion also depends upon of its frame of reference.

\section{Analysis of wave motion (it is a type of periodic motion) within a relative frames:}

A wave motion in which cycles are repeating after the same interval of time with the same distance between them.

\subsection{In wave equation c:}

\section{Where, $\quad c=$ wavelength $\times$ frequency}

Wavelength $(\lambda)$ : It is the length of cycles in a wave motion.

Frequency (f): The number of repeating cycles per unit time in a wave motion. 
Time period (T): The time required to create a complete one cycle in a wave motion. It is also reciprocal of the frequency.

Let's take a wave source which creates a wave in nature. We use two observer's ' $A$ ' and 'B' to measured wave velocity ' $v$ ' and wave equation ' $c$ ' in two different frames of reference. (Figure 3.1)

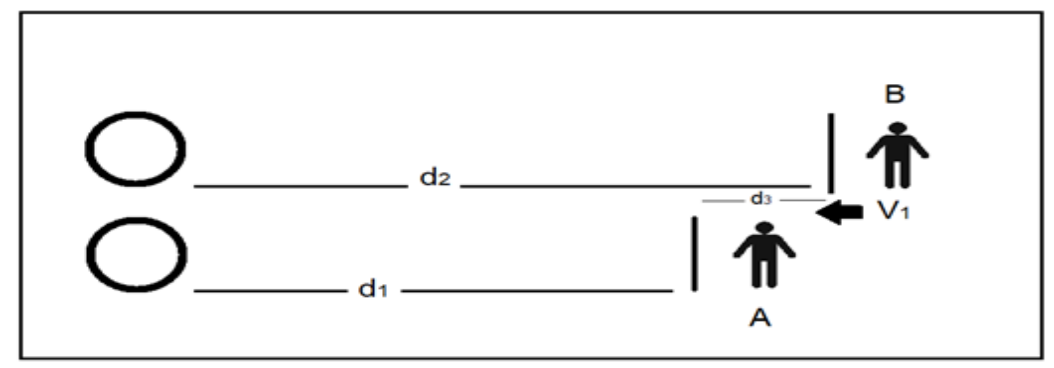

Figure 3.1

\subsection{In observer ' $A$ ' frame of reference or $1^{\text {st }}$ frame of reference:}

Consider an observer ' $A$ ' at rest and create a frame of reference to observe a wave motion. This is an observer ' $A$ ' frame of reference or $1^{\text {st }}$ frame of reference. Observer ' $A$ ' observed the wave source at rest in its frame of reference and observer ' $\mathrm{B}$ ' travel with velocity ' $\mathrm{V}_{1}$ ' in the negative $\mathrm{x}$-direction. The distance between observer ' $\mathrm{A}$ ' and observer ' $B$ ' is ' $\mathrm{d}_{3}$ '.

Consider a wave source which is producing 10 cycles in 1 second in nature. Observer ' $A$ ' take the reference distance $d_{1}$ to measure the velocity of a wave. Observer ' $A$ ' also counting cycles in wave motion and measuring a distance between these cycles in wave motion for wave equation $\mathrm{c}$.

Note: We can't draw a several of cycles produced by the source of a sound wave or the source of a light wave in nature. That's why we consider a wave source which is producing 10 cycles in 1 second for calculation of a velocity of a wave and wave equation $\mathrm{c}$.

Total number of cycles produced by a wave source in 1 second $(f)=10$ cycles per second

$$
\begin{aligned}
& \mathrm{d}_{1}=10 \text { meter } \\
& \mathrm{d}_{2}=15 \text { meter } \\
& \therefore \mathrm{d}_{3}=\left|\mathrm{d}_{1}-\mathrm{d}_{2}\right|=5 \text { meter } \\
& \therefore \mathrm{V}_{3}=\mathrm{d}_{3} / \mathrm{t}=5 \mathrm{~m} / \mathrm{s}
\end{aligned}
$$

\subsection{1. $1^{\text {st }}$ we measuring the velocity of a wave in observer ' $A$ ' frame of reference:}

A. $\mathrm{t}=\mathbf{0}$ second, (Figure 3.2)

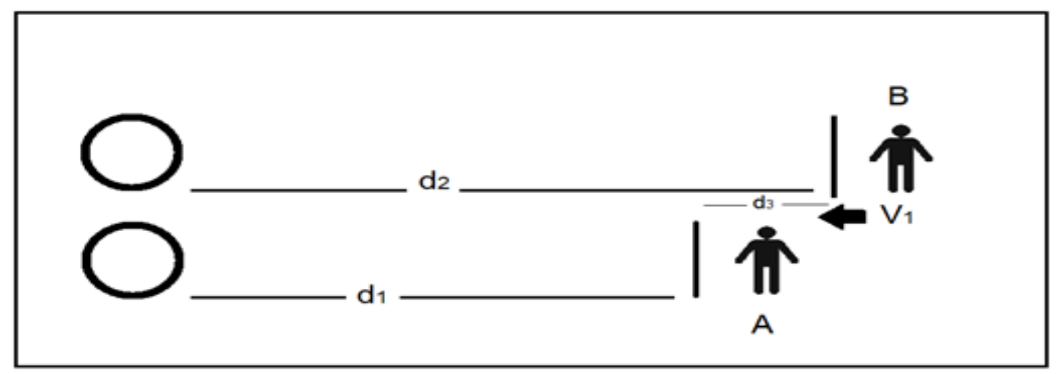

Figure 3.2

Numbers of cycles produced by the wave source $=0$,

Distance between observer ' $A$ ' and ' $B$ ' $=d_{3}$, 
B. $\mathbf{t}=\mathbf{0 . 1}$ second, (Figure 3.3)

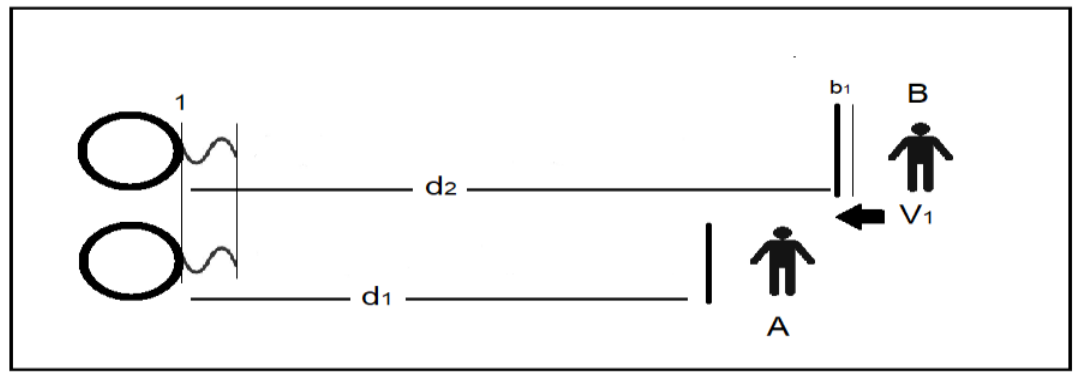

Figure 3.3

Numbers of cycles produced by the wave source $=1$,

Distance between observer ' $A$ ' and ' $\mathrm{B}$ ' $=\mathrm{d}_{3}-\mathrm{V}_{1} \times 0.1$,

C. $t=0.2$ second, (Figure 3.4)

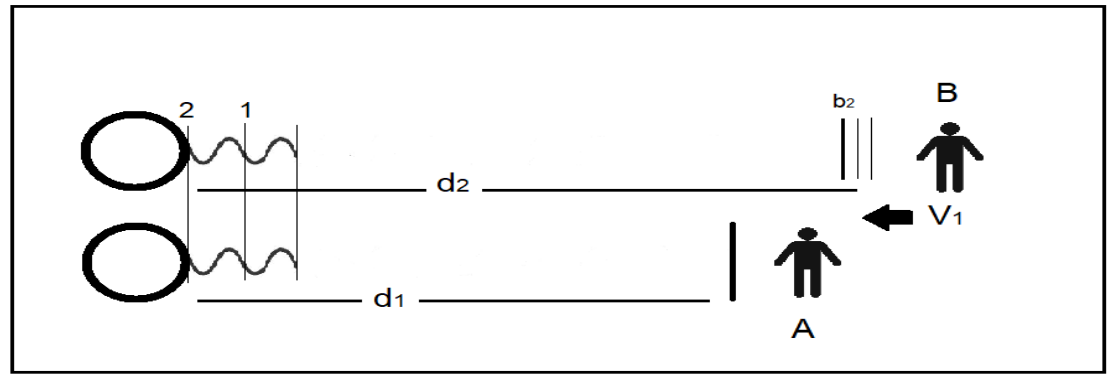

Figure 3.4

Numbers of cycles produced by the wave source $=2$,

Distance between observer ' $A$ ' and ' $\mathrm{B}$ ' $=\mathrm{d}_{3}-\mathrm{V}_{1} \times 0.2$,

D. $t=0.3$ second, (Figure 3.5)

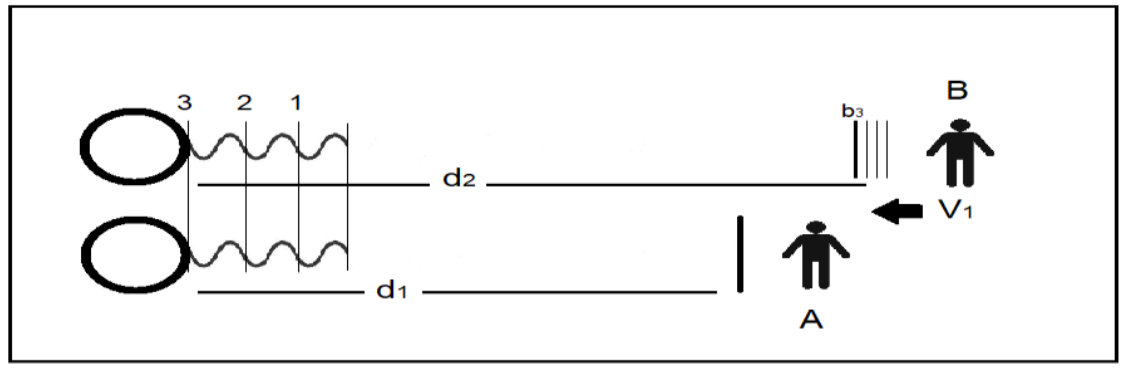

Figure 3.5

Numbers of cycles produced by the wave source $=3$,

Distance between observer ' $A$ ' and ' $B$ ' $=d_{3}-\overleftarrow{V_{1}} \times 0.3$,

And

E. $t=1.0$ second, (Figure 3.6)

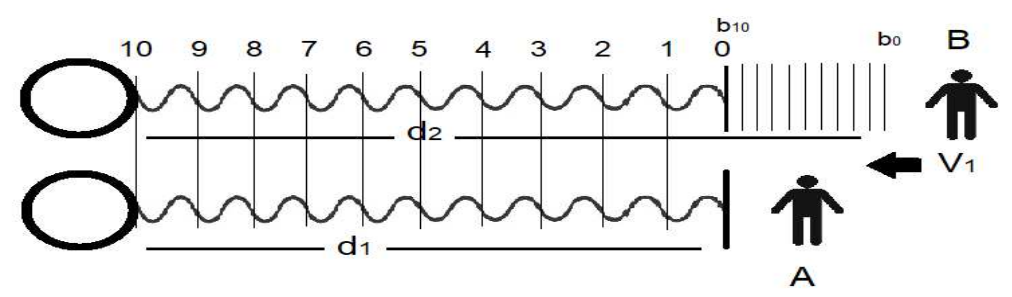


Numbers of cycles produced by the wave source $=10$,

Distance between observer ' $\mathrm{A}$ ' and ' $\mathrm{B}$ ' $=\mathrm{d}_{3}-\overline{\mathrm{V}_{1}} \times 1.0=0$

$$
\left(\mathrm{d}_{1}=\mathrm{d}_{2}\right)
$$

Note: At time $t=1.0$, both starts receiving a cycles of wave motion.

That means a wave is covered the distance $d_{1}$ in $1^{\text {st }}$ frame of reference.

Observer ' $A$ ' observed:

$$
\begin{array}{rlrl}
\text { Velocity of wave ' } \mathrm{V}_{\mathrm{a}} \text { ' } & =\frac{\text { distance }}{\text { Time }} & \frac{\mathrm{d}_{1}}{\mathrm{t}} \\
\overrightarrow{\mathrm{V}_{\mathrm{a}}}=\frac{10}{1} & =10 \mathrm{~m} / \mathrm{s}
\end{array}
$$$$
\text { (where } \mathrm{d}_{1}=10 \text { meter) }
$$

4.2.2. Let's count cycles of wave motion received by observer ' $A$ ' in 1 second and position of an observer ' $B$ ' in observer ' $A$ ' frame of reference:

F. $\mathrm{t}=1.1$ seconds, (Figure 3.7)

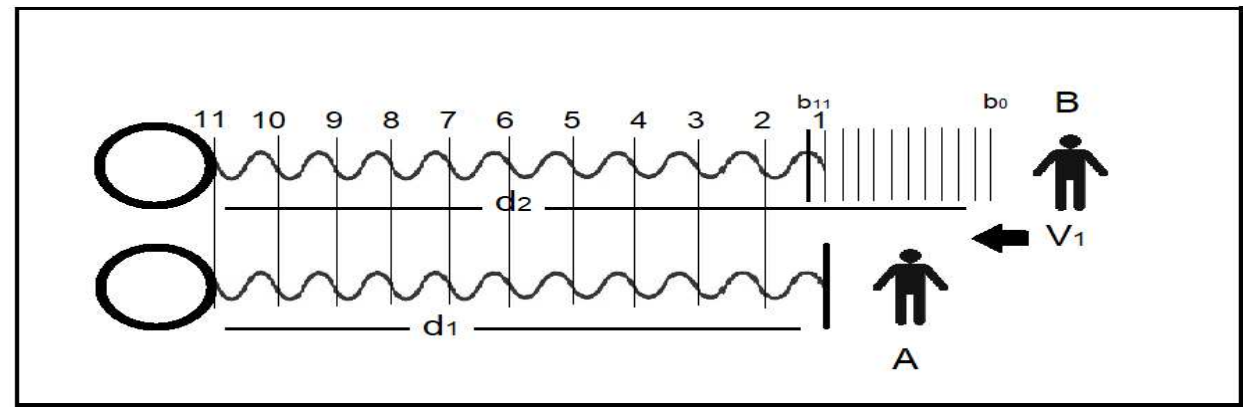

Figure 3.7

Number of cycles received by observer ' $A$ ' $=1$

Distance between observer ' $\mathrm{A}$ ' and ' $\mathrm{B}$ ' $\stackrel{4}{=} \overline{\mathrm{V}_{1}} \times 0.1=0.5 \mathrm{~m}$

Wavelength $(\lambda)=1 \mathrm{~m}$

But

Observer ' $\mathrm{B}$ ' received $1^{\text {st }}$ cycles of a wave $=1.066666 \ldots$ second

(same in both the frames)

And

G. $\mathbf{t}=\mathbf{2 . 0}$ seconds, (Figure 3.8)

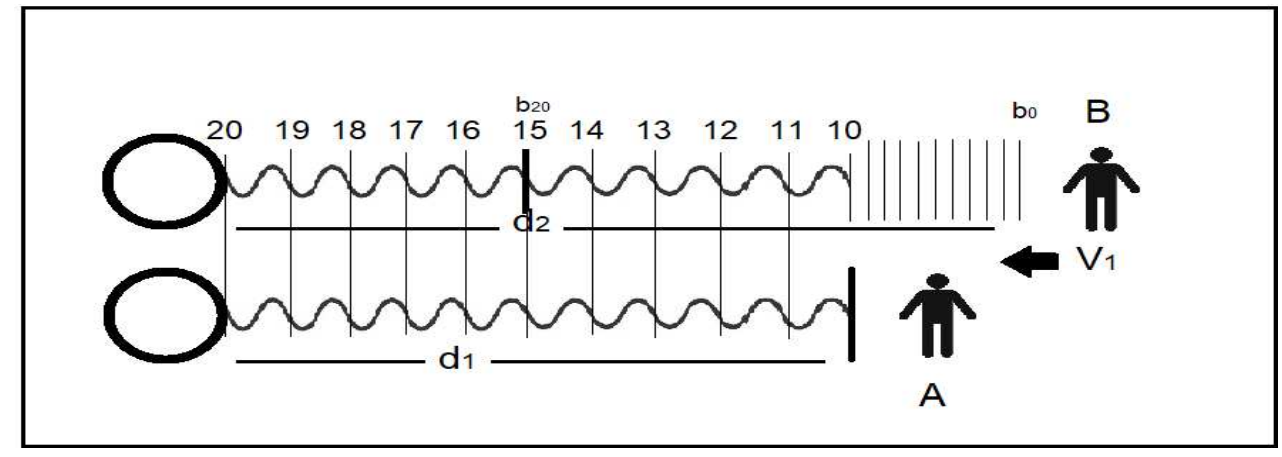

Figure 3.8 
Number of cycles received by observer ' $A$ ' $=10$

Distance between observer ' $A$ ' and ' $B$ ' $\stackrel{4}{=} \mathrm{V}_{1} \times 1.0=5 \mathrm{~m}$

Wavelength $(\lambda)=1 \mathrm{~m}$

\section{Important:}

Number of cycles received by observer 'B' $=15$

But

The wavelength $(\lambda)$ for observer ' $B$ ' according to observer ' $A$ ' or in observer ' $A$ ' frame is $=0.66666 \ldots \mathrm{m}$

In observer ' $A$ ' frame: Observer ' $B$ ' is traveling towards the wave source. Then frequency is increased but the wavelength of a wave will be decreased.

Observer ' $A$ ' calculate the wave equation $c$ in observer ' $A$ ' frame:

Wave equation ' $\mathrm{C}_{\mathrm{a} 1}$ ' $=$ length of cycle $\times$ numbers of cycles received by observer ' $\mathrm{A}$ ' in 1 second

$$
\mathrm{C}_{\mathrm{a} 1}=1 \times 10=10 \mathrm{~m} / \mathrm{s}
$$

\section{For Observer ' $B$ ' calculate the wave equation $c$ in observer ' $A$ ' frame:}

Wave equation ' $\mathrm{C}_{\mathrm{b} 1}$ ' $=$ length of cycle $\times$ numbers of cycles received by observer ' $\mathrm{B}$ ' in 1 second

$$
\mathrm{C}_{\mathrm{b} 1}=0.66666 \ldots \times 15=10 \mathrm{~m} / \mathrm{s}
$$

Where,

$$
\lambda f=\text { wavelength } \times \text { frequency }
$$

\section{Now, Comparison wave equation $\mathrm{c}$ for both observers with a wave velocity $\mathrm{v}$ in $\mathbf{1}^{\text {st }}$ frame:}

$$
\mathrm{V}_{\mathrm{a}}=\mathrm{C}_{\mathrm{a} 1}=\mathrm{C}_{\mathrm{b} 1}=10 \mathrm{~m} / \mathrm{s} \quad \text { (from eq. 3.1. eq. } 3.2 \text { and eq.3.3) }
$$

\subsection{In observer ' $B$ ' frame of reference or $2^{\text {nd }}$ frame of reference:}

In which observer ' $\mathrm{B}$ ' consider at rest and create a frame of reference to observe the wave motion. This is an observer ' $B$ ' frame of reference or $2^{\text {nd }}$ frame of reference.

But the wave source and observer ' $A$ ' are travel in positive $\mathrm{x}$-direction with same velocity ' $\mathrm{V}_{1}$ ' with respect to observer ' $\mathrm{B}$ '. That means for observer ' $\mathrm{B}$ ' considered at rest.

Total number of cycles produced by wave source in 1 second $(f)=10$ cycles per second (same like above)

$\mathrm{d}_{1}=10$ meter (same like above)

$\mathrm{d}_{2}=15$ meter (same like above)

$\therefore \mathrm{d}_{3}=\left|\mathrm{d}_{1}-\mathrm{d}_{2}\right|=5$ meter (same like above)

$\therefore \overrightarrow{\mathrm{V}_{3}}=\mathrm{d}_{3} / \mathrm{t}=5 \mathrm{~m} / \mathrm{s} \quad$ (same like above but the direction of a frame of reference has changed.)

1. $1^{\text {st }}$ we measuring the velocity of a wave in observer ' $B$ ' frame of reference:

(same like above)
A. $\mathbf{t}=\mathbf{0}$, (Figure 3.9) 


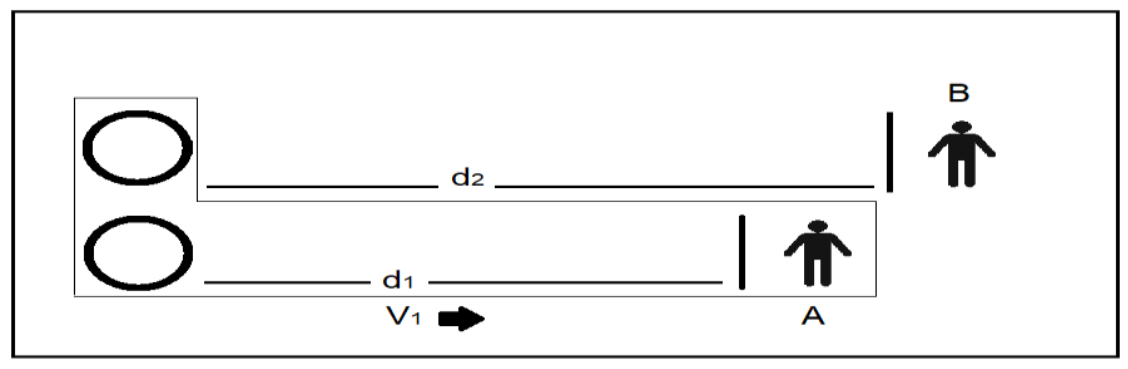

Figure 3.9

Number of cycles produced by the wave source $=0$,

Distance travel by wave source $=5 \times 0=0 \mathrm{~m}$,

Origin of $1^{\text {st }}$ event $=0 \mathrm{~m}$,

B. $\mathbf{t}=\mathbf{0 . 1},($ Figure 3.10)

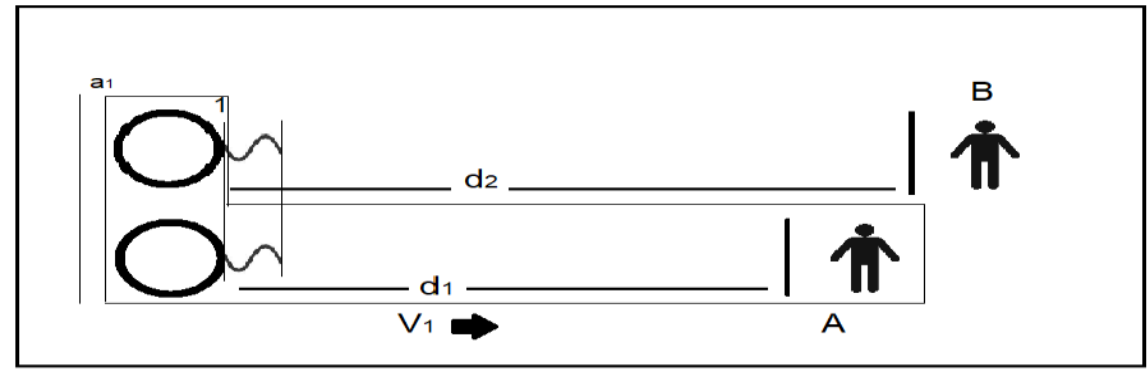

Figure 3.10

Number of cycles produced by the wave source $=1$,

Distance travel by wave source $=5 \times 0.1=0.5 \mathrm{~m}$,

Distance travel by $1^{\text {st }}$ event $=1.5 \times 1=1.5 \mathrm{~m}$,

Position of $1^{\text {st }}$ event $=0$ (origin distance) $+1.5=1.5 \mathrm{~m}$,

Origin of $2^{\text {nd }}$ event $=0.5 \mathrm{~m}$,

(distance travel by wave source)

C. $\mathbf{t}=\mathbf{0 . 2}$, (Figure 3.11)

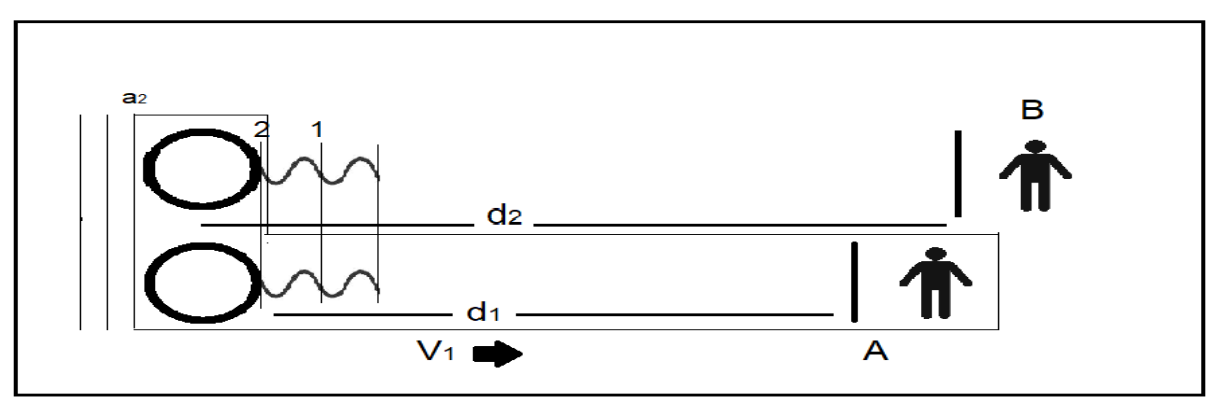

Figure 3.11

Number of cycles produced by the wave source $=2$,

Distance travel by wave source $=5 \times 0.2=1.0 \mathrm{~m}$,

Distance travel by $1^{\text {st }}$ event $=1.5 \times 2=3.0 \mathrm{~m}$,

Position of $1^{\text {st }}$ event $=0$ (origin distance $)+3.0=3.0 \mathrm{~m}$,

Distance travel by $2^{\text {nd }}$ event $=1.5 \times 1=1.5 \mathrm{~m}$,

Position of $2^{\text {nd }}$ event $=0.5$ (origin distance) $+1.5=2.0 \mathrm{~m}$,

Origin of $3^{\text {rd }}$ event $=1.0 \mathrm{~m}$, (distance travel by wave source)

D. $\mathbf{t}=\mathbf{0 . 3}$, (Figure 3.12) 


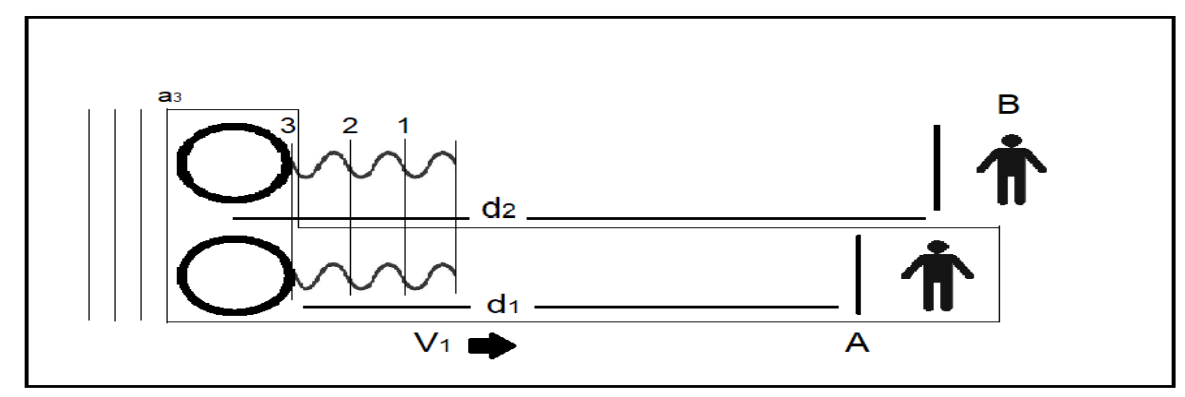

Figure 3.12

Number of cycles produced by the wave source $=3$,

Distance travel by wave source $=5 \times 0.3=1.5 \mathrm{~m}$,

Distance travel by $1^{\text {st }}$ event $=1.5 \times 3=4.5 \mathrm{~m}$,

Position of $1^{\text {st }}$ event $=0$ (origin distance $)+4.5=4.5 \mathrm{~m}$,

Distance travel by $2^{\text {nd }}$ event $=1.5 \times 2=3.0 \mathrm{~m}$,

Position of $2^{\text {nd }}$ event $=0.5$ (origin distance) $+3.0=3.5 \mathrm{~m}$,

Distance travel by $3^{\text {rd }}$ event $=1.5 \times 1=1.5 \mathrm{~m}$,

Position of $3^{\text {rd }}$ event $=1.0$ (origin distance) $+1.5=2.5$

Origin of $4^{\text {th }}$ event $=1.5 \mathrm{~m}$,

(distance travel by wave source)

E. $t=1.0$, (Figure 3.13)

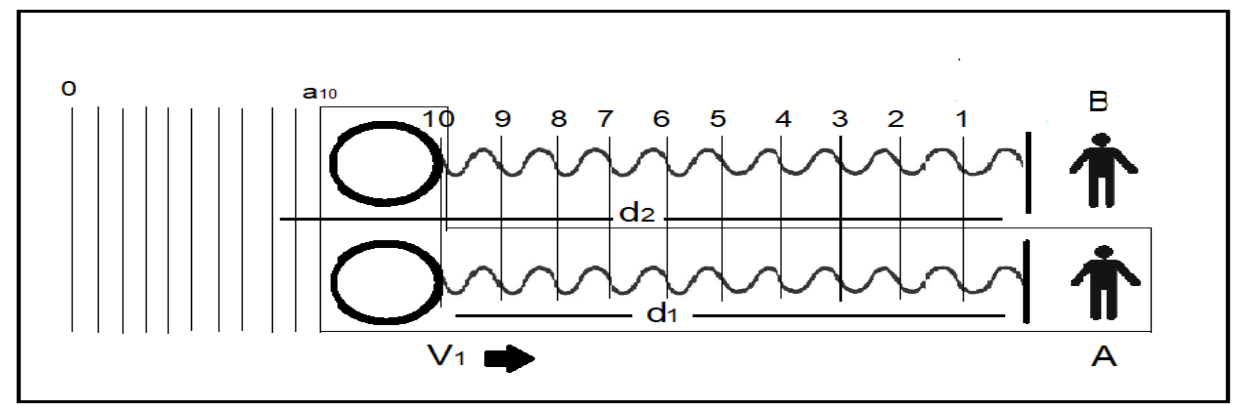

Figure 3.13

Number of cycles produced by the wave source $=10$,

Distance travel by wave source $=5 \times 1.0=5 \mathrm{~m}$,

Distance travel by $1^{\text {st }}$ event $=1.5 \times 10=15 \mathrm{~m}$,

Position of $1^{\text {st }}$ event $=0$ (origin distance) $+15=15 \mathrm{~m}$,

Distance travel by $2^{\text {nd }}$ event $=1.5 \times 9=13.5 \mathrm{~m}$,

Position of $2^{\text {nd }}$ event $=0.5$ (origin distance) $+13.5=14 \mathrm{~m}$,

Distance travel by $3^{\text {rd }}$ event $=1.5 \times 8=12 \mathrm{~m}$,

Position of $3^{\text {rd }}$ event $=1.0$ (origin distance) $+12=13$

Note: At time $t=1.0$, both starts receiving cycles of a wave motion.

(Same like above)

That means a wave is covered the distance $d_{2}$ in $2^{\text {nd }}$ frame of reference.

Observer 'B' observed:

$\begin{array}{cc}\text { Velocity of wave ' } \mathrm{V}_{\mathrm{b}} \text { ' }=\frac{\text { distance }}{\text { Time }} & \frac{\mathrm{d}_{2}}{\mathrm{t}} \\ \overrightarrow{\mathrm{V}_{\mathrm{b}}=\frac{15}{1}} & =15 \mathrm{~m} / \mathrm{s}\end{array}$

(where $\mathrm{d}_{2}=15$ meter) 
2. Let's count cycles of wave motion received by observer ' $B$ ' in 1 second and position of an observer ' $A$ ' in observer ' $B$ ' frame of reference:

F. $t=1.066666 \ldots$ seconds, (Figure 3.14)

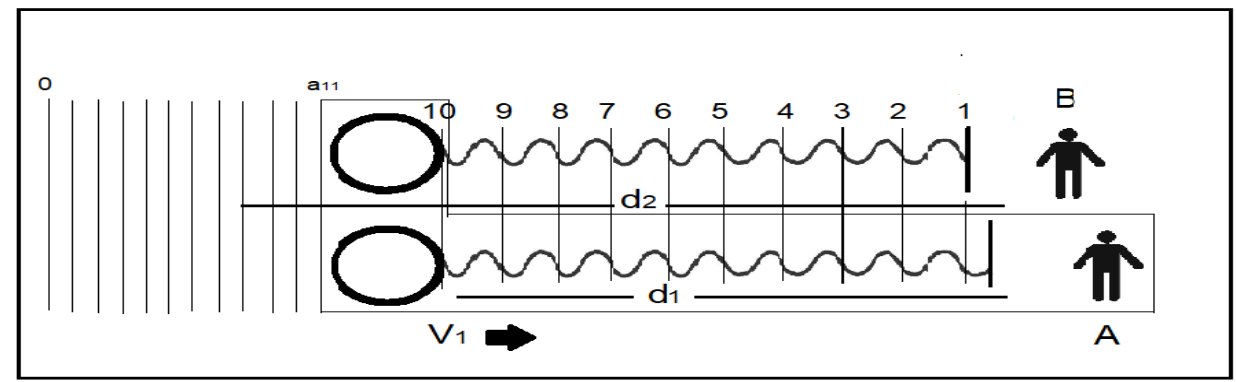

Figure 3.14

Number of cycles received by observer ' $\mathrm{B}$ ' $=1$

Distance between observer ' $B$ ' to ' $A$ ' $=\overrightarrow{V_{1}} \times 0.1=0.5 \mathrm{~m}$

Wavelength $(\lambda)=1 \mathrm{~m}$

But

Observer ' $A$ ' received $1^{\text {st }}$ cycles of a wave $=1.1$ seconds

(same in both the frames)

And

G. $\mathbf{t}=\mathbf{2 . 0}$ seconds, (Figure 3.15)

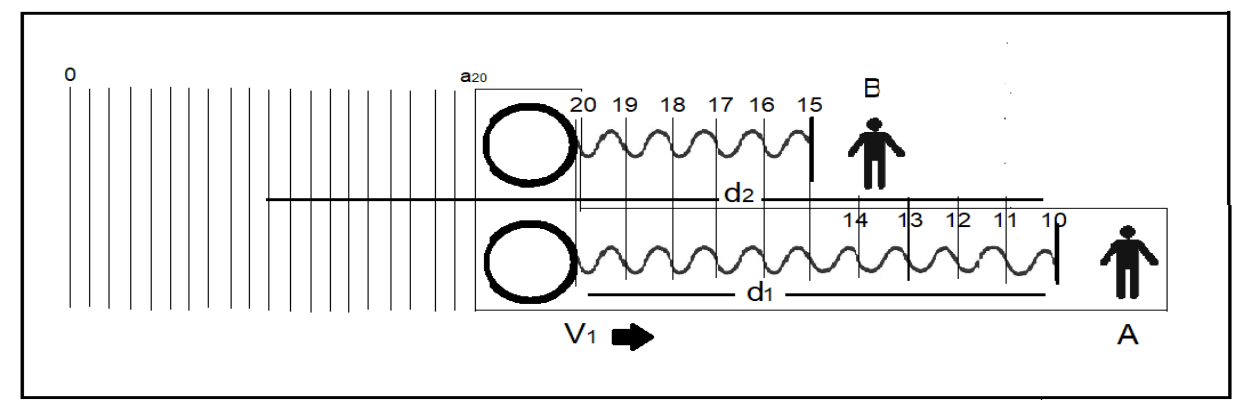

Figure 3.15

Number of cycles received by observer ' $\mathrm{B}$ ' $=15$

Distance between observer ' $B$ ' to ' $A$ ' $=\overrightarrow{V_{1}} \times 1.0=5 \mathrm{~m}$

Wavelength $(\lambda)=1 \mathrm{~m}$

\section{Important:}

Number of cycles received by observer ' $A$ ' $=10$

The wavelength $(\lambda)$ for observer ' $A$ ' according to observer ' $B$ ' and in observer ' $B$ ' frame is $=1.5 \mathrm{~m}$

In observer ' $B$ ' frame: Observer ' $A$ ' is traveling away the wave source. Then frequency is decreased but the wavelength of a wave will be increased.

\section{Observer ' $B$ ' calculate the wave equation c:}

Wave equation ' $\mathrm{C}_{\mathrm{b} 2}$ ' $=$ length of cycle $\times$ numbers of cycles received by observer ' $\mathrm{B}$ ' in 1 second

$$
\mathrm{C}_{\mathrm{b} 2}=1 \times 15=15 \lambda \mathrm{f}
$$

For Observer ' $A$ ' calculate the wave equation $c$ in an observer ' $A$ ' frame:

Wave equation ' $\mathrm{C}_{\mathrm{a} 2}$ ' $=$ length of cycle $\times$ numbers of cycles received by observer ' $A$ ' in 1 second

$$
\mathrm{C}_{\mathrm{a} 2}=1.5 \times 10=15 \lambda \mathrm{f}
$$


Now, Comparison wave equation $\mathrm{c}$ for both observers with a wave velocity $\mathrm{v}$ in $2^{\text {nd }}$ frame:

$$
\mathrm{V}_{\mathrm{b}}=\mathrm{C}_{\mathrm{a} 2}=\mathrm{C}_{\mathrm{b} 2}=15 \mathrm{~m} / \mathrm{s} \quad \text { (from eq. 3.5, eq.3.6 and eq. 3.7) }
$$

Where, $\lambda f=$ wavelength $\times$ frequency

Now, analyze all the equations:

1. Compare velocity of wave ' $v$ ' in both frames.

$$
\overrightarrow{\mathrm{V}_{\mathrm{a}}} \neq \overrightarrow{\mathrm{V}_{\mathrm{b}}}
$$

(from eq. 3.4 and eq. 3.8)

$10 \mathrm{~m} / \mathrm{s} \neq 15 \mathrm{~m} / \mathrm{s}$

$\therefore$ A velocity of a wave depends upon its frame of reference. Therefore, the velocity is not the same in all inertial frame of reference.

2. Compare the frequency received by both the observers is the same in both frames:

For observer ' $A$ ': $\mathrm{f}_{\mathrm{a} 1}=\mathrm{f}_{\mathrm{a} 2}=10$ cycles

$\therefore$ observer ' $A$ ' received 10 cycles of a wave per second in nature.

For observer ' $\mathrm{B}$ ': $\mathrm{f}_{\mathrm{b} 1}=\mathrm{f}_{\mathrm{b} 2}=15$ cycles

$\therefore$ observer ' $\mathrm{B}$ ' received 15 cycles of a wave per second in nature.

$\therefore$ The time takes for any events to happen the same for all observers in nature. That means time is an absolute quantity in nature.

3. Compare the wavelength measured by both the observers in both the frames:

In $1^{\text {st }}$ frame:

A wavelength measured by observer ' $A$ ' $=1 \mathrm{~m}$

A wavelength for observer ' $\mathrm{B}$ ' according to observer ' $\mathrm{A}$ ' $=0.66666 \ldots \mathrm{m}$

In $2^{\text {nd }}$ frame:

A wavelength measured by observer ' $\mathrm{B}$ ' $=1 \mathrm{~m}$

A wavelength for observer ' $A$ ' according to observer ' $B$ ' $=1.5 \mathrm{~m}$

$\therefore$ :Displacement of any motion always depends upon its reference frame. This is the reason the velocity of any motion is changed with respect to frames. Because the time takes for any events to happen the same for all observers in nature.

\subsection{Analyze most famous Ives-Stilwell experiments (1938-1941) on the basis of wave equation c:}

Herbert E. Ives and G. R. Stilwell performed experiments and calculated wave equation c of a light wave. They used two detectors for representing observer ' $A$ ' and Observer ' $B$ ' respectively and the light source placed between them [19] [20].

Case 1: The light source is rest with respect to both the observers. (Figure 3.16) 


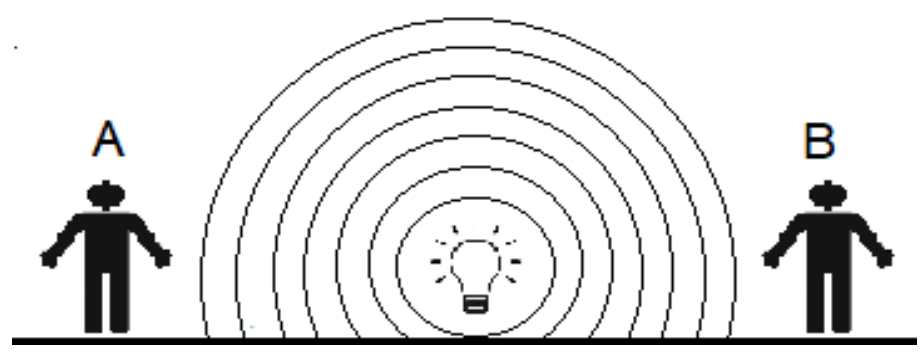

Figure 3.16

Wavelength for both the observers are the same $\left(\lambda_{0}\right)=4861 \mathrm{~A}^{0}$

And frequency for both the observers are also the same $\left(\mathrm{f}_{0}\right)=61715.696359 \times 10^{10} \mathrm{~Hz}$

Wave equation $\mathrm{c}$ for both the observers $=4861 \times 10^{10} \times 61715.696359 \times 10^{10}=3 \times 10^{8} \mathrm{~m} / \mathrm{s}$

(eq. 3.10)

Case 2: The light source is moving with velocity $\mathrm{V}_{\mathrm{s}}=0.005 \mathrm{C}$ towards the observer ' $\mathrm{B}$ '. (Figure 3.17$)\left(\mathrm{c}=3 \times 10^{8}\right.$ $\mathrm{m} / \mathrm{s}$ )

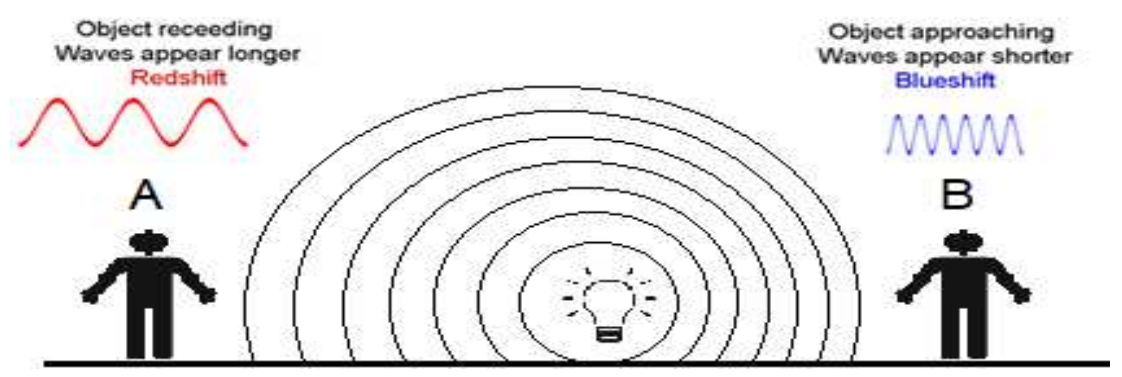

Figure 3.17

Observer ' $\mathrm{A}$ ' observed: wavelength $\left(\lambda_{\mathrm{a}}\right)=4885.18 \mathrm{~A}^{0}$

$$
\begin{aligned}
& \text { frequency }\left(\mathrm{f}_{\mathrm{a}}\right)=61410.224393 \times 10^{10} \mathrm{~Hz} \\
& \text { wave equation } \mathrm{C}_{\mathrm{a}}=3 \times 10^{8} \mathrm{~m} / \mathrm{s}
\end{aligned}
$$

Observer 'B' observed: wavelength $\left(\lambda_{\mathrm{b}}\right)=4836.94 \mathrm{~A}^{0}$

$$
\begin{gathered}
\text { frequency }\left(\mathrm{f}_{\mathrm{b}}\right)=62022.683763 \times 10^{10} \mathrm{~Hz} \\
\text { wave equation } \mathrm{C}_{\mathrm{b}}=3 \times 10^{8} \mathrm{~m} / \mathrm{s}
\end{gathered}
$$

Now, Compared both the cases and we found that both the observers at rest with each other in both cases. There nothing has any relative frame for observation. In both cases, you got the only frame for observation.

$$
\therefore \mathrm{C}=\mathrm{C}_{\mathrm{a}}=\mathrm{C}_{\mathrm{b}}
$$

(from eq. 3.10, eq. 3.11 and eq. 3.12)

That's why, we got the same velocity of light wave in both the cases.

\subsection{All experiments in which the light source observed by rotating observers or vice versa and calculate wave equation c:}

All those experiments in which the light source observed by rotating observers or vice versa and got wave equation $\mathrm{c}$ is the same. There they observed those experiments and calculated wave equation $\mathrm{c}$ in only one frame. That is the earth ground frame. There nothing has any relative frame for calculation. That's like in $\mathbf{4 . 2}$ and $\mathbf{4 . 3}$ wave events observation.

\section{Remember:}

The number of cycles in wave motion observed by observers is the same for all observers in relative frames. But displacement of motion depends upon its frame.

$>$ A velocity of any motion does not change until unbalanced force act upon it or if we compare with its relative frames. 


\section{Why is it difficult to understand?}

This is too simple when we see the definition of both the ' $c$ ' and ' $v$ ' which are different method with each other and also the mathematical equation for both the ' $c$ ' and ' $v$ ' is also different with each other. Where ' $v$ ' is displacement upon time and wave equation ' $c$ ' is an observer observed a wave motion per second and calculated the wave equation c.

\section{$2^{\text {nd }}$ mistake:}

All those experiments in which physicists found wave equation $\mathrm{c}$ is constant that observed in only one frame. That is the ground frame. An observer moving with respect to the ground frame or at rest with respect to the ground frame. There is nothing that has any relative frame for observation. This is the 2 nd and most complicated mistakes to create an illusion in physics. This had easily solved if we didn't do the 3rd mistake.

\section{Conclusion:}

- "A wave equation c = velocity of a wave", when we observed in only one frame. That means there is no another moving frame for comparison.

- Both observers received the same number of cycles at the same time in relative frames.

- But the wavelength of a wave is different in relative frames.

- The time takes for any events to happen are same for all observer in nature.

\section{The Michelson-Morley Experiment:}

This is the most famous, easy and best experiment to understand the law of physics works the same in all inertial frames. Because frames are not an absolute quantity in nature. The reference frame is created by an observer for measuring the displacement of a motion that happens in nature to its frame. This experiments also shown the beauty of physics. Why are we saying this sentence? Let's see ...

In the $19^{\text {th }}$ century, Physicists believes, a light wave required medium for its propagation and it is known as "luminiferous ether". In 1887, Albert A. Michelson and Edward W. Morley has performed an experiment to detect this "ether medium". They used a device called interferometer in which a light source is emitting a light wave which split with beam splitter into two beams which are travel perpendicular to each other [4].

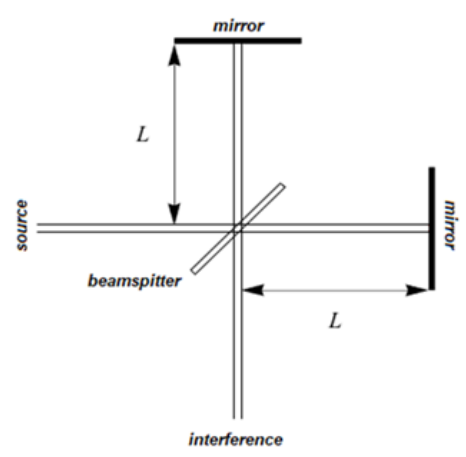

Figure 4.1 Demonstration of interferometer

After leaving the splitter, the beams travelled out to the ends of long arms placed at the same distance from the splitter, where they were reflected in the middle by small mirrors and producing a constructive or destructive interference pattern, recombined on the far side of the splitter to screen [4]. (Figure 4.1) 


\subsection{Let's see the concept of Michelson-Morley experiment:}

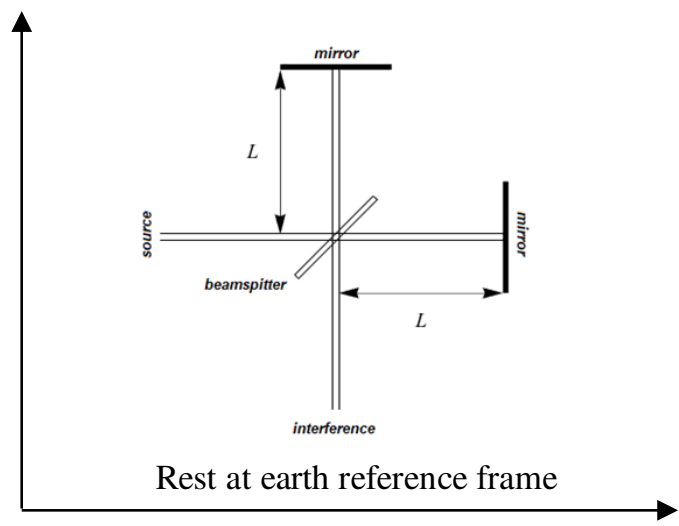

Figure 4.2

Generally, we have seen all events on earth in the earth reference frame. Michelson-Morley was set this experiment in the earth reference frame and observed contractive and distractive interference patterns on a screen. (Figure 4.2)

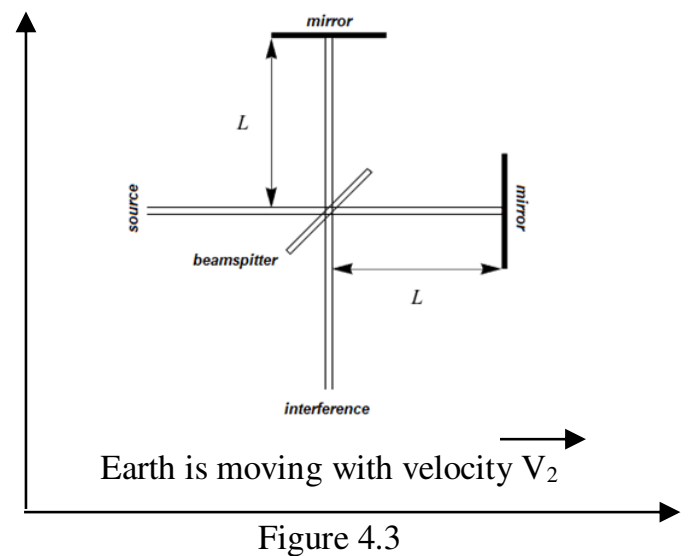

The Earth is in motion with respect to the sun. Then they assumed if the same experiment will be observed in the sun reference frame. Then the interference pattern will be changed because a speed of light wave is constant in all inertial frames. (Figure 4.3)

We already know the displacement of all motion is depends upon its frame. Therefore the velocity varies with one frame to another frame.

Consider two observers, observer ' $A$ ' is rest with respect to Experimental setup created at earth and observer ' $B$ ' is in motion with respect to observer ' $A$ '. This is a whole experiment is at rest with respect to observer 'A' and observed constructive or destructive interference pattern on the screen. If another observer ' $B$ ' observed the same experiment, but he is travel in uniform velocity with respect to observer ' $A$ ' and this experiment or vice versa. Then the interference pattern will change?

The Newton's $\mathbf{1}^{\text {st }}$ law of motion: "Every object will remain at rest or in uniform motion in a straight line unless an unbalanced force act upon it." [2]

\section{$3^{\text {rd }}$ mistake:}

Note, if something changes somewhere then any kind of force is responsible for it. Only for viewing position change of the observer, then will the result be changed? In Michelson-Morley experiment (and subsequent similar experiments), why they want to change in a light interference pattern? And what they got "Null Result". It could be easily solved when we understand the $1^{\text {st }}$ and $2^{\text {nd }}$ mistakes of in a wave motion or if someone had solved these experiments according to its relative frame mathematics, he would have found these mistakes too. But few physicists gave imagination-based solution to solve these experiments. This is the $3^{\text {rd }}$ mistake which is led to the creation of various hypothesis theories in physics. 


\subsection{Analysis a velocity of light within the sun reference frame:}

(i) A velocity of light in the earth reference frame in all directions is the same as that is ' $\mathrm{V}_{1}$ '. Now the case is the earth is travelling with velocity ' $\mathrm{V}_{2}$ ' in positive $\mathrm{x}$-direction with respect to the sun or relative observer ' $\mathrm{B}$ '. Therefore, a light wave from source to the beam splitter is ' $\mathrm{V}_{1}$ ' travel in positive $\mathrm{x}$ - direction in the earth reference frame. (Figure 4.4)

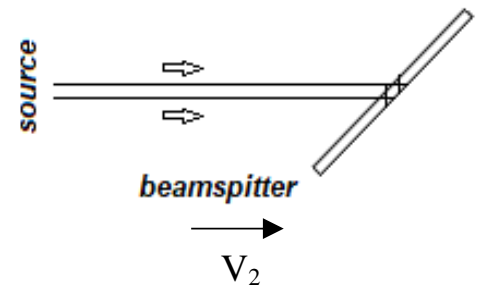

Figure 4.4

Then observer ' $\mathrm{B}$ ' observed velocity of light ' $\mathrm{V}$ ' ' is

Eq. $\overrightarrow{\mathrm{V}_{3}}=\overrightarrow{\mathrm{V}_{1}}+\overrightarrow{\mathrm{V}_{2}}$

(velocity of light in the sun reference frame)

(ii) A Light wave travels from beam slitter to front mirror and distance between them on the earth reference frame is ' $\mathrm{L}$ ' and Earth travel the distance ' $\mathrm{d}$ ' in $\mathrm{x}$ - direction with respect to observer ' $\mathrm{B}$ ' in the same time ' $\mathrm{t}$ '. (Figure 4.5)

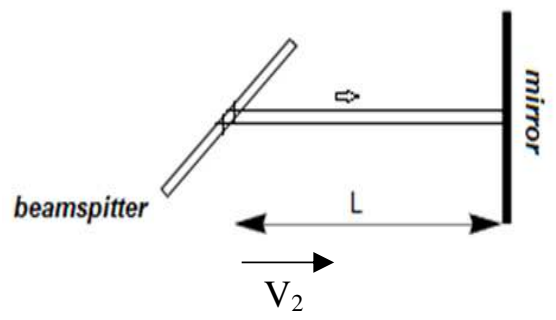

Figure 4.5

For observer ' $\mathrm{A}$ ' in earth reference frame is

Eq. $\mathrm{V}_{1} \times \mathrm{t}=\mathrm{L} \quad$ (distance reached by light in time ' $\mathrm{t}$ ' in earth reference frame)

For observer ' $\mathrm{B}$ ' in the reference frame is
Eq. $V_{3} \times t=L_{1} \quad$ (distance reached by a light wave in same time ' $t$ ' for observer ' $B$ ')
$\overrightarrow{\mathrm{V}_{3}}=\overrightarrow{\mathrm{V}_{1}}+\overrightarrow{\mathrm{V}_{2}}$
(velocity of light in the sun reference frame)
$\mathrm{L}_{1}=\mathrm{L}+\mathrm{d}$

(iii) A Light wave reflected from front mirror to beam splitter and Earth travel the distance ' $\mathrm{d}$ ' in $\mathrm{x}$ - direction with respect to observer 'B' at the same time ' $t$ '. (Figure 4.6)

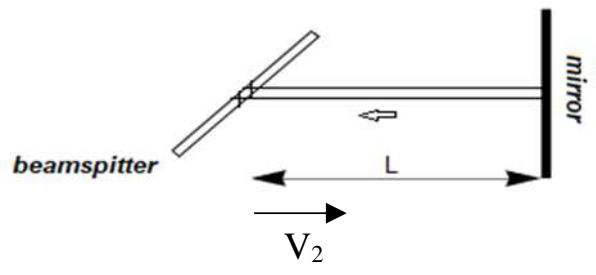

Figure 4.6 
For observer ' $\mathrm{A}$ ' in earth reference frame is

Eq. $\mathrm{V}_{1} \times \mathrm{t}=\mathrm{L}$

(distance reached by light photon in time ' $t$ ' in earth reference frame)

For observer ' $\mathrm{B}$ ' in the sun reference frame is

Eq. $\stackrel{\leftarrow \mathrm{V}_{3}}{\leftarrow} \overleftarrow{\mathrm{V}}_{1}-\overrightarrow{\mathrm{V}}_{2} \quad\left(\mathrm{~V}_{1}>>\mathrm{V}_{2}\right) \quad$ (velocity of light photon for observer ' $\mathrm{B}$ ')

$\mathrm{V}_{3} \times \mathrm{t}=\mathrm{L}_{1} \quad$ (distance reached by light photon in same time ' $\mathrm{t}$ ' for observer ' $\mathrm{B}$ ')

$\mathrm{L}_{1}=\mathrm{L}-\mathrm{d}$

(iv) A Light wave travel from beam splitter to a perpendicular mirror and in earth reference frame and return back and the Earth travel the distance ' $\mathrm{d}$ ' in $\mathrm{x}$ - direction with respect to observer ' $\mathrm{B}$ ' at the same time ' $\mathrm{t}$ '. (Figure 4.7)

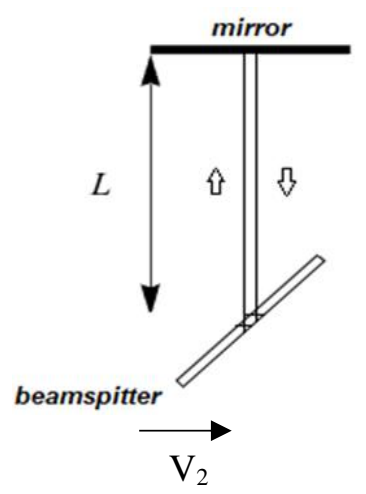

Figure 4.7

For observer ' $\mathrm{A}$ ' in earth reference frame is

Eq. $\mathrm{V}_{1} \times \mathrm{t}=\mathrm{L} \quad$ (distance reached by light photon in time ' $\mathrm{t}$ ' in earth reference frame)

For observer ' $\mathrm{B}$ ' in the sun reference frame is

Eq. $\left(\overrightarrow{\mathrm{V}_{3}}\right)^{2}=\overrightarrow{\left(\mathrm{V}_{1}\right)^{2}}+\overrightarrow{\left(\mathrm{V}_{2}\right)^{2}} \quad$ (velocity of light in the sun reference frame)

$\left(\mathrm{L}_{1}\right)^{2}=\mathrm{L}^{2}+\mathrm{d}^{2} \quad$ (distance reached by light in same time ' $\mathrm{t}$ ' for observer ' $\mathrm{B}$ ')

$\mathrm{V}_{3} \times \mathrm{t}=\mathrm{L}_{1}$

(v) Both light wave travel from beam splitter to the screen and Earth travel the distance ' $\mathrm{d}$ ' in $\mathrm{x}$ - direction with respect to observer ' $\mathrm{B}$ ' at the same time ' $\mathrm{t}$ '. (Figure 4.8)

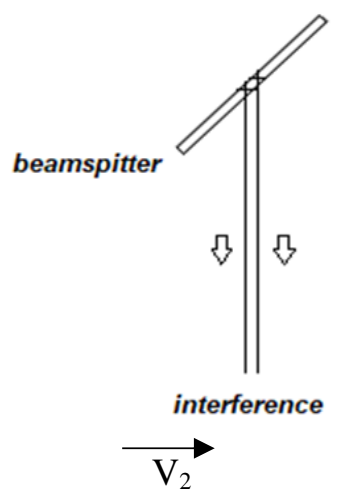

Figure 4.8

For observer ' $\mathrm{A}$ ' in earth reference frame is 
Eq. $\mathrm{V}_{1} \times \mathrm{t}=\mathrm{L}$

(distance reached by light in time ' $t$ ' in earth reference frame)

For observer ' $\mathrm{B}$ ' observed in the sun reference frame

Eq. $\overrightarrow{\left(\mathrm{V}_{3}\right)^{2}}=\overrightarrow{\left(\mathrm{V}_{1}\right)^{2}}+\overrightarrow{\left(\mathrm{V}_{2}\right)^{2}} \quad$ (velocity of a light wave in the sun reference frame)

$$
\begin{aligned}
& \left(\mathrm{L}_{1}\right)^{2}=\mathrm{L}^{2}+\mathrm{d}^{2} \quad \text { (distance reached by a light in same time ' } \mathrm{t} \text { ' for observer ' } \mathrm{B} \text { ') } \\
& \mathrm{V}_{3} \times \mathrm{t}=\mathrm{L}_{1}
\end{aligned}
$$

5.3 Observer ' $B$ ' observed a light wave traveling like this: (Figure 4.9)

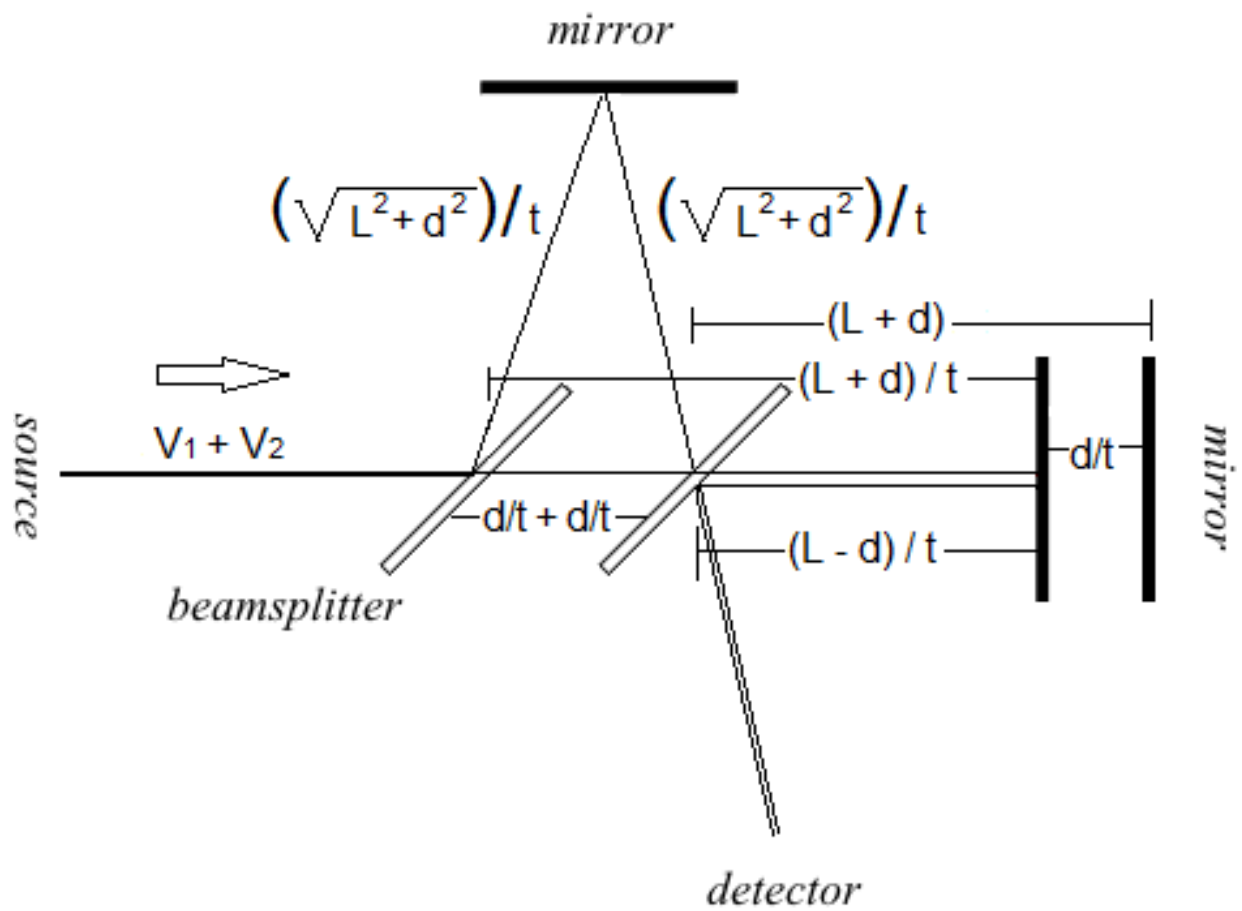

Figure 4.9

The time takes for light travels from the light source to the screen is the same for both the observers. But displacement of a motion depends upon its frame. Therefore the velocity of a light wave depends upon its frames. We can also verify this with the law of the conversation of momentum (before collision $=$ after collision). This is valid in all inertial frames. "This is the reason; the law of physics is the same for all inertial reference frame (i.e. non-accelerating reference frame)" [2] [7].

\section{Overall:}

Michelson-Morley has taken the same displacement of light wave for both the frames. Because they considered a velocity of light is constant in all inertial frames. This is the reason the mathematical calculation results and observation results were always different and created a mystery [4].

Not only Michelson-Morley, but this is also the list of subsequent similar experiments their results:

\begin{tabular}{|c|c|l|c|c|c|c|c|c|}
\hline $\begin{array}{c}\text { Sr. } \\
\text { No. }\end{array}$ & Name & Year & $\begin{array}{c}\text { Arm } \\
\text { length } \\
\text { (meters) }\end{array}$ & $\begin{array}{c}\text { Fringe shift } \\
\text { expected }\end{array}$ & $\begin{array}{c}\text { Fringe shift } \\
\text { measured }\end{array}$ & Ratio & $\begin{array}{c}\text { Experimental } \\
\text { Resolution }\end{array}$ & $\begin{array}{c}\text { Null } \\
\text { result }\end{array}$ \\
\hline 1. & Michelson [4] & 1881 & 1.2 & 0.04 & $\leq 0.02$ & 2 & 0.02 & Yes \\
\hline 2. & $\begin{array}{c}\text { Michelson and } \\
\text { Morley [5] }\end{array}$ & 1887 & 11.0 & 0.4 & $\begin{array}{l}<0.02 \\
\text { or } \leq 0.01\end{array}$ & 40 & 0.01 & Yes \\
\hline 3. & Morley and Miller [8] & $1902-1904$ & 32.2 & 1.13 & $\leq 0.015$ & 80 & 0.015 & Yes \\
\hline 4. & Miller [9] & 1921 & 32.0 & 1.12 & $\leq 0.08$ & 15 & Unclear & Unclear \\
\hline 5. & Miller [9] & $1923-1924$ & 32.0 & 1.12 & $\leq 0.03$ & 40 & 0.03 & Yes \\
\hline
\end{tabular}




\begin{tabular}{|c|c|l|c|c|l|c|c|c|}
\hline 6. & Miller (sunlight) [9] & 1924 & 32.0 & 1.12 & $\leq 0.014$ & 80 & 0.014 & Yes \\
\hline 7. & $\begin{array}{c}\text { Tomaschek (star } \\
\text { light) [10] }\end{array}$ & 1924 & 8.6 & 0.3 & $\leq 0.02$ & 15 & 0.02 & Yes \\
\hline 8. & Miller [9] [11] & $1925-1926$ & 32.0 & 1.12 & $\leq 0.088$ & 13 & Unclear & Unclear \\
\hline 9. & Kennedy [12] & 1926 & 2.0 & 0.07 & $\leq 0.002$ & 35 & 0.002 & Yes \\
\hline 10. & Illingworth [13] & 1927 & 2.0 & 0.07 & $\leq 0.0004$ & 75 & 0.0004 & Yes \\
\hline 11. & Piccard \& Stahel [14] & 1926 & 2.8 & 0.13 & $\leq 0.006$ & 20 & 0.006 & Yes \\
\hline 12. & Piccard \& Stahel [15] & 1927 & 2.8 & 0.13 & $\leq 0.0002$ & 185 & 0.0007 & Yes \\
\hline 13. & Piccard \& Stahel [16] & 1927 & 2.8 & 0.13 & $\leq 0.0003$ & 185 & 0.0007 & Yes \\
\hline 14. & Michelson et al. [17] & 1929 & 25.9 & 0.9 & $\leq 0.01$ & 90 & 0.01 & Yes \\
\hline 15. & Joos [18] & 1930 & 21.0 & 0.75 & $\leq 0.002$ & 375 & 0.002 & Yes \\
\hline
\end{tabular}

All people who experimented based on relative observation. They got either null results or the results of incorrect interpretations. That means there were no changes in the light interference pattern or results of incorrect interpretations.

\section{Discussion:}

This could have been easily solved if they go by a perfect mathematical expression. But few physicists gave additional mathematics and explanation to fix these differences. Finally, they bend our mathematical scales (i.e. Length Contraction and Time dilation) to give a solution [6] [7].

"This is the reason; the law of physics is same for all inertial reference frame (i.e. non-accelerating reference frame)" [2] [7]. Because the frame is created by an observer. Therefore the frame is not an absolute quantity in nature.

\section{Here, the name of some physicists which found a velocity of a light wave is not a constant in all inertial frames and proved with the help their own experiments, GPS data or mathematics:}

NASA scientist Daniel Y. Gezari has performed a comprehensive literature search and found that none of the five photonic effects has ever been observed in nature which predicted by the special theory of relativity [21] and also Gezari's lunar laser test found that the speed of light is not a constant in all inertial frame [22]. Physicist Xinhang Shen has modified the Fizeau Experiment and proved the speed of light is not constant [23]. Physicist Koshun Suto has proved that the speed of light is not constant in all inertial frame using the Correlation between to Two Pair-Generated Photons [24]. Physicist Masanori Sato has proved that the group velocity is observed differently due to the velocity of the observer by experimental evidence of The Sagnac effect in GPS data [25]. Physicist Stephan Gift has shown invalidate the principle of light speed constancy through One-way Speed of Light Using Interplanetary Tracking Technology [26]. Bahram Kalhor, Farzaneh Mehrparvar and Behnam Kalhor has compared multi-dimensional energy-momentum equation and relativistic energy- momentum equation and roved the special theory of relativity is wrong [27]. Physicist Yuan Zhong Zhang has shown the loopholes in the special theory of relativity through a physical test of theMS transformations is a test of the two-way speed of light, but not of the one-way speed of light [28]. Researchers Guochang Xu and Yan Xu found a speed of light is not a constant for all observers by GPS [29]. In 2018, Scientists Neil Ashby, Thomas E. Parker \& Bijunath R. Patla published a null test of general relativity based on a long-term comparison of atomic transition frequencies [30]. Physicist Antonio Luigi Paolilli has shown the problems related with the special theory of relativity [31]. Physicist Robert J. Buenker has shown a deduction of relativistic length variations based on tests using a Cryogenic Optical Resonator [32].

There are also many physicists which they proved the theory of relativity is wrong by mathematically and experimentally. But these experiments are ignored by mainstream physicists only for these lists of experiments which we discussed above where STR explained the results by bend mathematical scales [7].

\section{A device which can draw a graphical representation of an even in its relative frame:}

Let's take two graph pages for two observers 'A' and 'B' and one carbonic page place between these two pages. Now, you will start to move $2^{\text {nd }}$ page in any direction with velocity and starts graphical representation of any event on 
the $1^{\text {st }}$ page. The $1^{\text {st }}$ graph page represents $1^{\text {st }}$ observer reference frame and $2^{\text {nd }}$ graph page is represent $2^{\text {nd }}$ observer reference frame. You will see the velocity an event is changing with respect to the direction and velocity of the $2^{\text {nd }}$ page. It is easy to calculate two or more of an event in relative frame.

\section{Example:}

Case 1: $1^{\text {st }}$ page at rest with respect to you and $2^{\text {nd }}$ page is moving in the negative x-direction with velocity $10 \mathrm{~cm} / \mathrm{s}$. Now, draw a $10 \mathrm{~cm}$ straight line on the $1^{\text {st }}$ page in the positive $\mathrm{x}$-direction in 1 second.

\section{Observe:}

The length of the straight line:

$$
\begin{aligned}
& \text { In } 1^{\text {st }} \text { page }=10 \mathrm{~cm} \quad \text { (you drew) } \\
& \text { In } 2^{\text {nd }} \text { page }=10 \mathrm{~cm}+\text { velocity of } 2^{\text {nd }} \text { page } \\
& =10 \mathrm{~cm}+10 \mathrm{~cm}=20 \mathrm{~cm}
\end{aligned}
$$

Velocity of the straight line:

$$
\begin{aligned}
& \text { In } 1^{\text {st }} \text { page }=\frac{10}{1}=10 \mathrm{~cm} / \mathrm{s} \\
& \text { In } 2^{\text {nd }} \text { page }=\frac{20}{1}=20 \mathrm{~cm} / \mathrm{s}
\end{aligned}
$$

Remember: The time takes for any events to happen are same for all observer in nature. But the displacement of a motion changes with respect to its frames. Therefore the velocity of a motion changes in all relative frames.

With the help of this device, we can find the value of ' $\mathrm{v}$ ' and 'c' of a motion in it's a relative frames. Like Doppler Effect for a sound wave, Michelson-Morley and subsequent similar experiments, Relativistic Doppler Effect for a light wave, Sagnac Effect and all the motion which we are measured in a relative frames.

The remaining problems in which we found that time dilation effects will solve in my next journal.

In the $19^{\text {th }}$ century, Physicists believes, a light wave required medium for its propagation. This equation still remains.

\section{Conclusion:}

In this study, we found that the displacement of any motion always depends upon its frames. The frame created by an observer for measuring the displacement of any motion in its frame is not an absolute quantity in nature. The time takes for any events to happen the same for all observers in nature. Therefore the velocity of any motion also depends upon its frame of reference. There is no special condition present in nature where a velocity of a light wave is invariance in all inertial frames.

All those experiments in which physicists found wave equation $\mathrm{c}$ is constant that observed in only one frame. That is the ground frame. An observer moving with respect to the ground frame or at rest with respect to the ground frame. There is nothing that has any relative frame for observation.

The Michelson-Morley experiments in which they have taken the same displacement of light wave for both the frames. Because they considered a velocity of light is constant in all inertial frames. This is the reason the mathematical calculation and observation results were always different and created a mystery. But few physicists gave additional mathematics and explanation to fix these differences. Finally, they bend our mathematical scales (i.e. Length Contraction and Time dilation) to give a solution. Their imagination-based theories deceive by producing a misleading or false impression of reality. 
This study also reveals that space in-universe is similar like Galilean Co-ordination and Galilean Transformation (transform between the Co-ordinates of two reference frames). Length, Time, Mass, Temperature etc. are our scales. Therefore the concepts such as Length contraction and Time dilation effects have never existed in nature.

\section{Conflicts of Interest:}

The authors declares no conflicts of interest regarding the publication of this paper.

\section{References:}

[1] Galilei, Galileo (1638I). Leiden: Elsevier. pp. 191-196.

[2] Newton, Sir Isaac; Machin, John (1729). Principia. 1 (1729 translation Ed.). p. 19.

[3] Maxwell, J. Clerk (1 January 1865). "A Dynamical Theory of the Electromagnetic Field". Philosophical Transactions of the Royal Society of London. 155: 459-512.

[4] Michelson, Albert A. (1881). "The Relative Motion of the Earth and the Luminiferous Ether". American Journal of Science. 22 (128): 120-129. https://doi.org/10.2475\%2Fajs.s3-22.128.120

[5] Michelson, Albert A.; Morley, Edward W. (1887). "On the Relative Motion of the Earth and the Luminiferous Ether". American Journal of Science. 34 (203): 333-345. https://doi.org/10.2475\%2Fajs.s3-34.203.333

[6] Lorentz, Hendrik Antoon (1892), "The Relative Motion of the Earth and the Aether", Zittingsverlag Akad. V. Wet., 1: 74-79

[7] Albert Einstein (1905) "Zur Elektrodynamik bewegter Körper", Annalen der Physik. 17: 891; English translation On the Electrodynamics of Moving Bodies by George Barker Jeffery and Wilfrid Perrett (1923).

[8] Morley, Edward W. \& Miller, Dayton C. (1905). "Report of an experiment to detect the Fitzgerald-Lorentz Effect". Proceedings of the American Academy of Arts and Sciences. XLI (12): 321-8. https://doi.org/10.2307\%2F20022071

[9] Miller, Dayton C. (1925). "Ether-Drift Experiments at Mount Wilson". Proceedings of the National Academy of Sciences. 11 (6): 306-314. https://doi.org/10.1073\%2Fpnas.11.6.306

[10] Tomaschek, R. (1924). "Über das Verhalten des Lichtes außerirdischer Lichtquellen". Annalen der Physik. 378 (1): $105-126$. https://doi.org/10.1002\%2Fandp.19243780107

[11] Miller, Dayton C. (1933). "The Ether-Drift Experiment and the Determination of the Absolute Motion of the Earth". Reviews of Modern Physics. 5 (3): 203-242. https://doi.org/10.1103\%2FRevModPhys.5.203

[12] Kennedy, Roy J. (1926). "A Refinement of the Michelson-Morley Experiment". Proceedings of the National Academy of Sciences. 12 (11): 621-629. https://doi.org/10.1073\%2Fpnas.12.11.621

[13] Illingworth, K. K. (1927). "A Repetition of the Michelson-Morley Experiment Using Kennedy's Refinement" (PDF). https://doi.org/10.1103\%2FPhysRev.30.692

[14] Piccard, A.; Stahel, E. (1926). "L'expérience de Michelson, réalisée en ballon libre". Comptes Rendus. 183 (7): 420 -421.

[15] Piccard, A.; Stahel, E. (1927). "Nouveaux résultats obtenus par l'expérience de Michelson". Comptes Rendus. $184: 152$.

[16] Piccard, A.; Stahel, E. (1927). "L'absence du vent d'éther au Rigi". Comptes Rendus. 184: 1198-1200.

[17] Michelson, A. A.; Pease, F. G.; Pearson, F. (1929). "Results of repetition of the Michelson-Morley experiment". Journal of the Optical Society of America. 18 (3): 181. https://doi.org/10.1364\%2Fjosa.18.000181

[18] Joos, G. (1930). "Die Jenaer Wiederholung des Michelsonversuchs". Annalen der Physik. 399 (4): 385-407. https://doi.org/10.1002\%2Fandp.19303990402

[19] Ives, H. E.; Stilwell, G. R. (1938). "An experimental study of the rate of a moving atomic clock". Journal of the Optical Society of America. 28 (7): 215. https://doi.org/10.1364\%2FJOSA.28.000215

[20] Ives, H. E.; Stilwell, G. R. (1941). "An experimental study of the rate of a moving atomic clock. II". Journal of the Optical Society of America. 31 (5): 369. https://doi.org/10.1364\%2FJOSA.31.000369

[21] Daniel Y. Gezari, http://arxiv.org/ftp/arxiv/papers/0912/0912.3818.pdf

[22] Daniel Y. Gezari, http://arxiv.org/ftp/arxiv/papers/0912/0912.3934.pdf

[23] Xinhang Shen (2016), “Challenge to the Special Theory of Relativity”. Physics Essays 29 (1):142-148. https://doi.org/10.4006/0836-139829.1 .142

[24] Koshun Suto (2020), “A Violation of the Special Theory of Relativity Demonstrated Using the Correlation between Two Pair-Generated Photons". Journal of Applied Mathematics and Physics, 2020, 8, 1755-1766. https://doi.org/10.4236/jamp.2020.89132 
[25] Masanari Sato (2010), “The velocity of electromagnetic wave is observed differently depending on the observer's velocity”. Physics Essays 23, pp. 405-412(8). https://doi.org/10.4006/1.3452472

[26] Stephan Gift (2014), “One-way Speed of Light Using Interplanetary Tracking Technology”. Physical Science International Journal 4, Page 780-796. https://doi.org/10.9734/PSIJ/2014/7783

[27] Kalhor, Bahram; Mehrparvar, Farzaneh; kalhor, Behnam (2020): Is Einstein's special relativity wrong? Fig share. Preprint. https://doi.org/10.6084/m9.figshare.12585245.v4

[28] Yuan Zhong Zhang (1995), “Test theories of special relativity”. General Relativity and Gravitation 27, 475-493. https://doi.org/10.1007/BF02105074

[29] Guochang Xu and Yan Xu (2016), “GPS - Theory, Algorithms and Applications”. Springer-Verlag Berlin Heidelberg, https://doi.org/10.1007/978-3-662-50367-6

[30] Neil Ashby, Thomas E. Parker \& Bijunath R. Patla (2018), “A null test of general relativity based on a long-term comparison of atomic transition frequencies”. Nature Physics 14, 822-826. https://doi.org/10.1038/s41567-018-0156-2

[31] Antonio Luigi Paolilli (2020), "Superluminal Relativistic Phenomena in the Hypothesis of a Privileged Inertial System". International Journal of Innovation in Science and Mathematics 8, Issue 4, ISSN (Online): 2347-9051.

[32] Robert J Buenker (2020), “Deduction of relativistic length variations based on tests using a Cryogenic Optical Resonator”. Int J Nanomater Nanotechnol Nanomed 6(2): 016-020. https://dx.doi.org/10.17352/2455-3492.000035 